\title{
GROUP ACTIONS WITH TOPOLOGICALLY STABLE MEASURES
}

\author{
MEIHUA DONG ${ }^{1}$, SANGJIN KIM ${ }^{2}$, AND JIANDONG YIN ${ }^{3}$ \\ ${ }^{1,2}$ Department of Mathematics \\ Chungnam National University \\ Daejeon, 305-764, KOREA \\ ${ }^{3}$ Department of Mathematics \\ Nanchang University \\ Nanchang, 330-031, P.R. CHINA
}

\begin{abstract}
We prove that if an action $T$ of a finitely generated group $G$ on a compact metric space $X$ is measure expansive and has the measure shadowing property then it is measure topologically stable. This represents a measurable version of the main result in [4]. Moreover we prove that if $G$ is a finitely generated virtually nilpotent group and there exists $g \in G$ such that $T_{g}$ is expansive and has the invariant measure shadowing property then $T$ is invariant measure topologically stable. Finally we show that minimal actions approximated by periodic ones have no topologically stable measures.
\end{abstract}

AMS Subject Classification: 37C85, 54H20

Key Words: expansiveness, group action, pseudo-orbit tracing property, subshift of finite type, topological stability

Received: August 7, 2017; Accepted: December 29, 2017; Published: January 28, $2018 \quad$ doi: 10.12732/dsa.v27i1.10

Dynamic Publishers, Inc., Acad. Publishers, Ltd. https://acadsol.eu/dsa

\section{INTRODUCTION}

Walters [12] introduced the notion of topological stability, a kind of stability for homeomorphisms for which continuous pertubations are allowed, and proved that every expansive homeomorphism with the shadowing property on a compact metric space is topologically stable. Recently, Lee and Morales [6] obtained a measurable 
version of this result that any expansive measure with the shadowing property is topologically stable. This represents a further contribution to the study of expansive measures developed elsewhere in the recent literature $[2,3,8,11]$.

Very recently, Chung and Lee [4] introduced the notion of topological stability for finitely generated group actions, and Pilyugin et. al [7, 9, 10] introduced the notions of shadowing and invese shadowing for finitely generated group actions which are generalizations of those of topological stability, shadowing and inverse shadowing, respectively, for homeomorphisms on compact metric spaces.

In this paper, we consider a measure version of the dynamics of finitely generated group actions on compact metric spaces which were developed in $[4,7,9,10]$. More precisely, we introduce the notions of measure shadowing property and measure topological stability of group actions, and prove that if an action $T$ of a finitely generated group $G$ is measure expansive and has the measure shadowing property then it is measure topologically stable. Moreover we show that if $G$ is a finitely generated virtually nilpotent group and there exists $g \in G$ such that $T_{g}$ is expansive and has the invariant measure shadowing property then $T$ is invariant measure topologically stable. Finally we claim that minimal actions approximated by periodic ones have no topologically stable measures.

\section{PRELIMINARIES}

We round out the introduction with some notations that we will use in the paper. Let $G$ be a finitely generated group and $X$ be a compact metric space with a metric $d$. Let $\operatorname{Homeo}(X)$ be the space of all homeomorphisms of $X$. We denote by $\operatorname{Act}(G, X)$ the set of all continuous actions $T$ of $G$ on $X$, i.e., $T: G \times X \rightarrow X$ is a continuous map such that $T(e, x)=x$ and $T(g, T(h, x))=T(g h, x)$ for $x \in X$ and $g, h \in$ $G$, where $e$ is the identity element of $G$. For briefness, $T(g, x)$ will be denoted by $T_{g}(x)$. Let Homeo $(X)^{G}=\prod_{G} H o m e o(X)$ be the set of homeomorphisms from $G$ to $\operatorname{Homeo}(X)$ with the product topology. Then $\operatorname{Act}(G, X)$ can be considered as a subset of $H$ omeo $(X)^{G}$. Let $A$ be a symmetric finitely generating set of $G$, i.e., for any $a \in A, a^{-1} \in A$. If $A$ is a finintely generating set of $G$, then there always exists a symmetric finitely generating set containing $A$. Throughout the paper, a finitely generating set $A$ of $G$ implies a symmetric finitely generating set. We define a metric $d_{A}$ on $\operatorname{Act}(G, X)$ by

$$
d_{A}(T, S)=\sup \left\{d\left(T_{a} x, S_{a} x\right) \mid x \in X, a \in A\right\}
$$

for $T, S \in \operatorname{Act}(G, X)$. Then the topology on $\operatorname{Act}(G, X)$ induced by $d_{A}$ coincides with the product topology on $\operatorname{Act}(G, X)$ inherited from $\operatorname{Homeo}(X)^{G}$. Hence the space $\operatorname{Act}(G, X)$ is a separable complete metrizable topological space, and so a Baire space. 
Recall that the Borel $\sigma$-algebra of $X$ is the smallest $\sigma$-algebra generated by all open sets. Its elements will be referred to as Borelians of $X$. A Borel measure on $X$ is a $\sigma$-additive measure defined on the Borelians of $X$. All Borel measures $\mu$ will be assumed to be nontrivial (i.e., $\mu(X)>0$ ), and the set of all Borel measues on $X$ will be denoted by $\mathcal{M}(X)$. A point $x \in X$ is an atom of $\mu \in \mathcal{M}$ if $\mu(\{x\})>0$ for every $x \in X$. We say that $\mu \in \mathcal{M}(X)$ is nonatomic if it has no atoms, and the set of all nonatomic measures on $X$ will be deonted by $\mathcal{M}^{*}(X)$. In the sequel we proceed to extend the notions of expansivity, shadowing property and topological stability for group actions to Borel measures.

We say that $T \in A(G, X)$ is expansive if there is $c>0$ called an expansive constant of $T$ such that $d\left(T_{g} x, T_{g} y\right) \leq c$ for all $g \in G$ implies $x=y$. Equivalently, $\left.\Gamma_{c}^{T}(x)\right)=\{x\}$ for all $x \in X$, where $\Gamma_{c}^{T}(x)=\left\{y \in X \mid d\left(T_{g} x, T_{g} y\right) \leq c\right.$ for every $\left.g \in G\right\}$. A Borel measure $\mu \in \mathcal{M}(X)$ is said to be expansive with respect to a homeomorphism $f$ of $X$ if there is $\varepsilon>0$ such that $\mu\left(\Gamma_{\varepsilon}^{f}(x)\right)=0$ for every $x \in X$ (see [6]). By this motivation, we introduce a notion of expansive measure with respect to an action $T \in \operatorname{Act}(G, X)$.

Definition 2.1. For any Borel measure $\mu \in \mathcal{M}(X)$, an action $T \in \operatorname{Act}(G, X)$ is said to be $\mu$-expansive (or $\mu$ is expansive with respect to $T$ ) if there is $c>0$ such that $\mu\left(\Gamma_{c}^{T}(x)\right)=0$ for every $x \in X$. Moreover, we say that $T$ is measure expansive if $T$ is $\mu$-expansive for all $\mu \in \mathcal{M}^{*}(X)$.

It is clear that every expansive measure for a group action is nonatomic, and any nonatomic measure with repect to an expansive action $T \in \operatorname{Act}(G, X)$ is expansive.

If $T$ and $S$ are two continuous actions of $G$ on $X$ with $d_{A}(T, S)<\delta$, then the $S$ orbit $\left\{S_{g} x\right\}_{g \in G}$ of $x \in X$ is nearly a $T$-orbit in the sense that $d\left(T_{a} S_{g} x, S_{a g} x\right)<\delta$ for all $a \in A$ and $g \in G$. This observation motivates the following definition. Let $A$ be a finitely generating set of $G$ and $\delta>0$. A $\delta$-pseudo orbit of $T \in \operatorname{Act}(G, X)$ with respect to $A$ is a sequence $\left\{x_{g}\right\}_{g \in G}$ in $X$ such that $d\left(T_{a} x_{g}, x_{a g}\right)<\delta$ for all $a \in A, g \in G$. An action $T \in \operatorname{Act}(G, X)$ is said to have the shadowing property with respect to $A$ if for every $\varepsilon>0$, there exists $\delta>0$ such that any $\delta$-pseudo orbit $\left\{x_{g}\right\}_{g \in G}$ for $T$ with respect to $A$ is $\varepsilon$-traced by some point $x$ of $X$, that is, $d\left(T_{g} x, x_{g}\right)<\varepsilon$ for all $g \in G$.

Now we extend the notion of shadowing property of group actions to Borel measures. Given a subset $B$ of $X$, we say that a sequence $\left\{x_{g}\right\}_{g \in G}$ is through $B$ if $x_{e} \in B$.

Definition 2.2. Let $A$ be a finitely generating set of $G$ and $\mu \in \mathcal{M}(X)$. We say that an action $T \in A c t(G, X)$ has the $\mu$-shadowing property with respect to $A$ (or $\mu$ has the shadowing property with respect to $T$ and $A$ ) if for every $\varepsilon>0$ there are $\delta>0$ and a Borelian $B \subset X$ with $\mu(X \backslash B)=0$ such that every $\delta$-pseudo orbit $\left\{x_{g}\right\}_{g \in G}$ of $T$ with respect to $A$ through $B$ is $\varepsilon$-traced by some point $x \in X$, i.e., $d\left(T_{g} x, x_{g}\right)<\varepsilon$ 
for all $g \in G$. Moreover $T$ has the measure shadowing property with respect to $A$ if $T$ has the $\mu$-shadowing property with respect to $A$ for all $\mu \in \mathcal{M}^{*}(X)$.

It is clear that the definition of shadowing property of $T$ does not depend on the choice of a compatible metric $d$ on $X$. Furthermore, we can see that measure shadowing property of $T$ does not depend on the choice of a finitely generating set $A$ of $G$ as we can see in the following lemma.

Lemma 2.3. Let $A_{1}$ and $A_{2}$ be two finitely generating sets of $G$. An action $T \in$ $\operatorname{Act}(G, X)$ has the measure shadowing property with respect to $A_{1}$ if and only if it has the measure shadowing property with respect to $A_{2}$.

Proof. Assume that $T \in \operatorname{Act}(G, X)$ has the measure shadowing property with respect to $A_{1}$, and let $\mu \in \mathcal{M}(X)$. Then for any $\varepsilon>0$, by definition there exist $\delta>0$ and a Borelian $B \subset X$ with $\mu(X \backslash B)=0$ such that every $\delta$-pseudo orbit for $T$ with respect to $A_{1}$ through $B$ is $\varepsilon$-traced by some point of $X$. Put $m=\max _{a \in A_{1}} l_{A_{2}}(a)$, where $l_{A_{2}}$ is the word length metric on $G$ induced by $A_{2}$. Choose $\delta^{\prime}>0$ such that $m \delta^{\prime}<\delta$. Since $X$ is compact, $A_{1}$ and $A_{2}$ are finite and $T$ is continuous, there exists $\delta^{\prime \prime}>0$ such that for all $x, y \in X$ with $d(x, y)<\delta^{\prime \prime}$, one has $d\left(T_{g} x, T_{g} y\right)<\delta^{\prime}$ for every $g \in G$ with $l_{A_{2}}(g) \leq m$. Choose $0<\delta_{1}<\min \left\{\delta^{\prime}, \delta^{\prime \prime}\right\}$ such that for all $x, y \in X$ with $d(x, y)<\delta_{1}$, one has $d\left(T_{b^{-1}} x, T_{b^{-1}} y\right)<\delta^{\prime \prime}$ for every $b \in A_{2}$. It suffices to show that every $\delta_{1}$-pseudo orbit of $T$ with respect to $A_{2}$ through $B$ is a $\delta$-pseudo orbit of $T$ with respect to $A_{1}$ through $B$. Let $\left\{x_{g}\right\}_{g \in G}$ be a $\delta_{1}$-pseudo orbit for $T$ with respect to $A_{2}$ i.e., $d\left(T_{b} x_{g}, x_{b g}\right)<\delta_{1}$ for every $b \in A_{2}, g \in G$. For every $b \in A_{2}, g \in G$, since $d\left(x_{g}, T_{b} x_{b^{-1} g}\right)=d\left(x_{b b^{-1} g}, T_{b} x_{b^{-1} g}\right)<\delta_{1}$, one has

$$
d\left(T_{b^{-1}} x_{g}, T_{b^{-1}} T_{b} x_{b^{-1} g}\right)=d\left(T_{b^{-1}} x_{g}, x_{b^{-1} g}\right)<\delta^{\prime \prime} .
$$

Thus $d\left(T_{h} T_{b^{-1}} x_{g}, T_{h} x_{b^{-1} g}\right)=d\left(T_{h} T_{b} x_{g}, T_{h} x_{b g}\right)<\delta^{\prime}$ for every $b \in A_{2}, g \in G$ and $h \in G$ with $l_{A_{2}}(h) \leq m$. For any $a \in A_{1}$, we write $a$ as $b_{1} \cdots b_{l(a)}$ where $l(a)=$ $l_{A_{2}}(a), b_{i} \in A_{2}$. Then for any $a \in A_{1}, g \in G$, we have

$$
\begin{aligned}
d\left(T_{a} x_{g}, x_{a g}\right) & =d\left(T_{b_{1} \cdots b_{l(a)}} x_{g}, x_{b_{1} \cdots b_{l(a)} g}\right) \\
& \leq d\left(T_{b_{1} \cdots b_{l(a)}} x_{g}, T_{b_{1} \cdots b_{l(a)-1}} x_{b_{l(a)} g}\right) \\
& +d\left(T_{b_{1} \cdots b_{l(a)-1}} x_{b_{l(a)} g}, T_{b_{1} b_{2} \cdots b_{l(a)-2}} x_{b_{l(a)-1} b_{l(a)} g}\right) \\
& +\cdots+d\left(T_{b_{1} b_{2}} x_{b_{3} \cdots b_{l(a)} g}, T_{b_{1}} x_{b_{2} \cdots b_{l(a)} g}\right) \\
& +d\left(T_{b_{1}} x_{b_{2} \cdots b_{l(a)}}, x_{b_{1} \cdots b_{l(a)} g}\right) \\
& <(m-1) \delta^{\prime}+\delta_{1}<(m-1) \delta^{\prime}+\delta^{\prime}=m \delta^{\prime}<\delta .
\end{aligned}
$$

This means that $\left\{x_{g}\right\}_{g \in G}$ is a $\delta$-pseudo orbit of $T$ with respect to $A_{1}$ through $B$, and so completes the proof. 
Definition 2.4. We say that an action $T \in \operatorname{Act}(G, X)$ has the measure shadowing property if $T$ has the measure shadowing property with respect to $A$ for some finitely generating set of $G$.

It is clear that if an action $T \in \operatorname{Act}(G, X)$ has the shadowing property then it has the measure shadowing property, but the converse is not true in general.

Recently, Chung and lee [4] introduced the notion of topological stability for group actions as follows. Let $A$ be a finitely generating set of $G$. An action $T \in \operatorname{Act}(G, X)$ is said to be topologically stable with respect to $A$ if for every $\varepsilon>0$, there exists $\delta>0$ such that if $S$ is another continuous action of $G$ on $X$ with $d_{A}(T, S)<\delta$ then there exists a continuous map $f: X \rightarrow X$ satisfying $T_{g} \circ f=f \circ S_{g}$ for every $g \in G$ and $d\left(f, I d_{X}\right) \leq \varepsilon . T$ is said to be topologically stable if it is topologically stable with respect to $A$ for some finitely generating set $A$ of $G$.

To define topologically stable measure we will use some basic notations. Denote by $2^{X}$ the set formed by the subsets of $X$. A map $H: X \rightarrow 2^{X}$ will be referred to as a setvalued map of $X$. We define the domain of $H$ by $\operatorname{Dom}(H)=\{x \in X: H(x) \neq \emptyset\}$. We say that $H$ is compact-valued if $H(x)$ is compact for every $x \in X$. We write $d\left(H, I d_{X}\right)<\varepsilon$ for some $\varepsilon>0$ if $H(x) \subset B[x, \varepsilon]$ where $B[.,$.$] denotes the closed$ ball operation (note that such an inclusion is obvious for points $x \notin \operatorname{Dom}(H)$ ). A set-valued map $H$ of $X$ is upper semicontinuous if for every $x \in \operatorname{Dom}(H)$ and every neighborhood $O$ of $H(x)$ there is $\eta>0$ such that $H(y) \subset O$ whenever $y \in X$ with $d(x, y)<\eta$. With these terminologies, we introduce the notion of measure topological stability of group actions.

Definition 2.5. Let $A$ be a finitely generating set of $G$ and $T \in \operatorname{Act}(G, X)$. For any $\mu \in \mathcal{M}(X)$, we say that an action $T$ is $\mu$-topologically stable with respect to $A$ (or $\mu$ is topologically stable with respect to $T$ and $A$ ) if for every $\varepsilon>0$, there is $\delta>0$ such that for each continuous action $S$ of $G$ on $X$ with $d_{A}(T, S)<\delta$ there is an upper semicontinuous compact-valued map $H$ of $X$ with measurable domain such that $\mu(X \backslash \operatorname{Dom}(H))=0, \mu \circ H=0, d\left(H, I d_{X}\right) \leq \varepsilon$, and $T_{g} \circ H=H \circ S_{g}$ for $g \in G$. Moreover we say that $T$ is measure topologically stable if $T$ is $\mu$-topologically stable with respect to $A$ for all $\mu \in \mathcal{M}^{*}(X)$.

The definition of measure topological stability of $T \in \operatorname{Act}(G, X)$ is independent of the choice of generating sets of $G$ as we can see in the following lemma.

Lemma 2.6. Let $A_{1}$ and $A_{2}$ be finitely generating sets of $G$. An action $T \in$ $\operatorname{Act}(G, X)$ is measure topologically stable with respect to $A_{1}$ if any only if it is measure topologically stable with respect to $A_{2}$.

Proof. Suppose $T$ is measure topologically stable with respect to $A_{1}$. Then for any 
$\varepsilon>0$, there exists $\delta^{\prime}>0$ such that if $S$ is another continuous action of $G$ on $X$ with $d_{A_{1}}(T, S)<\delta^{\prime}$ then there is an upper semicontinuous compact-valued map $H$ of $X$ with measurable domain such that $\mu(X \backslash \operatorname{Dom}(H))=0, \mu \circ H=0, d\left(H, I d_{X}\right) \leq \varepsilon$, and $T_{g} \circ H=H \circ S_{g}$ for $g \in G$. It suffices to show that there exists $\delta>0$ such that for every $S \in \operatorname{Act}(G, X)$ if $d_{A_{2}}(T, S)<\delta$ then $d_{A_{1}}(T, S)<\delta^{\prime}$. Put $m=\max _{a \in A_{1}} l_{A_{2}}(a)$, where $l_{A_{2}}$ is the word length metric on $G$ induced by $A_{2}$. Choose $\delta_{1}>0$ such that $m \delta_{1}<\delta^{\prime}$. Since $X$ is compact and $T$ is continuous, there exists $\delta>0$ such that $d\left(T_{h} x, T_{h} y\right)<\delta_{1}$ for all $x, y \in X$ with $d(x, y)<\delta$ and for every $h \in G$ with $l_{A_{2}}(h) \leq m$. For any $a \in A_{1}$, we write $a$ as $b_{1} \cdots b_{l(a)}$, where $l(a)=l_{A_{2}}(a) \leq m, b_{i} \in A_{2}, i=1,2, \cdots, l(a)$. Then for every $S \in \operatorname{Act}(G, X)$ with $d_{A_{2}}(T, S)<\delta$, one has

$$
\begin{aligned}
d\left(T_{a} x, S_{a} x\right) & =d\left(T_{b_{1} \cdots b_{l(a)}} x, S_{b_{1} \cdots b_{l(a)}} x\right) \\
& \leq d\left(T_{b_{1} \cdots b_{l(a)-1}} T_{b_{l(a)}} x, T_{b_{1} \cdots b_{l(a)-1}} S_{b_{l(a)}} x\right) \\
& +d\left(T_{b_{1} \cdots b_{l(a)-2}} T_{b_{l(a)-1}} S_{b_{l(a)}} x, T_{b_{1} \cdots b_{l(a)-2}} S_{b_{l(a)-1}} S_{b_{l(a)}} x\right) \\
& +\cdots+d\left(T_{b_{1}} T b_{2} S_{b_{3} \cdots \cdots b_{l(a)}} x, T_{b_{1}} S_{b_{2}} S_{b_{3}} \cdots S_{l(a)} x\right) \\
& +d\left(T_{b_{1}} S_{b_{2} \cdots b_{l(a)}} x, S_{b_{1} \cdots b_{l(a)}} x\right) \\
& <m \delta_{1}<\delta^{\prime} .
\end{aligned}
$$

This means that $d_{A}(T, S)<\delta^{\prime}$, and so completes the proof.

Finally we introduce the notion of measure topological stability for group actions as follows.

Definition 2.7. An action $T \in \operatorname{Act}(G, X)$ is measure topologically stable if it is measure topologically stable with respect to some finitely generating set $A$ of $G$.

\section{ACTIONS WITH TOPOLOGICALLY STABLE MEASURES}

In the previous section, we extended the concept of topological stability for group actions to Borel measures. First, we see that if an action $T \in \operatorname{Act}(G, X)$ is topologically stable, then it is measure topologically stable.

Theorem 3.1. If an action $T \in \operatorname{Act}(G, X)$ is topologically stable, then it is measure topologically stable.

Proof. Suppose an action $T \in \operatorname{Act}(G, X)$ is topologically stable and $\mu \in \mathcal{M}^{*}(X)$. Let $A$ be a finitely generating set of $G$. Fix $\varepsilon>0$ and $\delta>0$ as in the definition of topological stability for $T$. Take $S \in \operatorname{Act}(G, X)$ with $d_{A}(T, S)<\delta$. Then there is a continuous map $h: X \rightarrow X$ with $T_{g} \circ h=h \circ S_{g}$ for all $g \in G$, and $d\left(h, I d_{X}\right) \leq \varepsilon$. Define a map $H$ of $X$ by $H(x)=\{h(x) \mid x \in X\}$. Then we have $\operatorname{Dom}(H)=X$, 
and $H$ is compact-valued. Since $h$ is continuous, $H$ is upper semicontinuous and $\mu(X \backslash \operatorname{Dom}(H))=0$. Since $\mu$ is nonatomic, $\mu(H(x))=\mu(\{h(x)\})=0$ for $x \in X$. Moreover, we have $d(H(x), x)=d(h(x), x) \leq \varepsilon$ for all $x \in X$. Since $T_{g} \circ h=h \circ S_{g}$, we get $T_{g} \circ H=H \circ S_{g}$, and so completes the proof.

As we have mentioned before, every expansive measure for group action is nonatomic, but topologically stable measure need not be nonatomic in general. In fact, let $f_{0}$ be a homeomorphism on $[0,1]$ given by

$$
f_{0}(t)=\left\{\begin{array}{cc}
\frac{1}{2} t, & 0 \leq t \leq \frac{1}{4} \\
\frac{3}{2} t-\frac{1}{4}, & \frac{1}{4} \leq t \leq \frac{3}{4}, \\
\frac{1}{2} t+\frac{1}{2}, & \frac{3}{4} \leq t \leq 1 .
\end{array}\right.
$$

Put $p_{n}=1 / 2^{n}, p_{-n}=1-1 / 2^{n}$ for each $n \in \mathbb{N}$. Consider a homeomorphism $f$ on $S^{1}=[0,1] / \sim$ which has the following properties: $f(0)=0$ and

$$
f(x)=\left\{\begin{array}{l}
p_{n+1}+\left(1 / 2^{n+1}\right) f_{0}\left(2^{n+1}\left(x-p_{n+1}\right)\right), p_{n+1} \leq x \leq p_{n} \\
p_{-n}+\left(1 / 2^{n+1}\right) f_{0}\left(2^{n+1}\left(x-p_{-n}\right)\right), p_{-n} \leq x \leq p_{-n-1}
\end{array}\right.
$$

where $n \in \mathbb{N}$. Then Yano [13] proved that $f$ is not topologically stable, but it has the shadowing property. Moreover we have

$$
\Omega(f)=\left\{p_{n} \mid n \in \mathbb{Z}\right\} \cup\left\{\frac{p_{n}+p_{n+1}}{2} \mid n \in \mathbb{Z}\right\} \cup\{0\} .
$$

By Colloary 4.5 in [6], we see that the Dirac measure $\mu_{p}\left(p \in S^{1} \backslash \Omega(f)\right)$ is topologically stable with respect to $f$.

Now we prove that if an action of a finitely generated group on a compact metric space is measure expansive and has the measure shadowing property then it is measure topologically stable. This extends the main result of Theorem 2.8 in [4] to Borel measures. The techiques of the proof resembles that of Theorem 3.1 in [6], but we will give a detailed proof here for safety.

Theorem 3.2. Any measure expansive action $T \in \operatorname{Act}(G, X)$ with the measure shadowing property is measure topologically stable. More precisely, if $T$ is $\mu$-expansive and has the $\mu$-shadowing property then it is $\mu$-topologically stable for all $\mu \in \mathcal{M}^{*}(X)$.

Proof. Let $A$ be a finitely generating set of $G$ and $\mu \in \mathcal{M}^{*}(X)$. Suppose that $T \in \operatorname{Act}(G, X)$ is $\mu$-expansive and has the $\mu$-shadowing property. Let $c$ be a $\mu$ expansive constant of $T$. Take $\varepsilon>0$ and $0<\varepsilon^{\prime}<\min \left\{\frac{c}{2}, \varepsilon\right\}$. For this $\varepsilon^{\prime}$, we let $\delta$ and $B$ be given by the definition of the $\mu$-shadowing property of $T$. Let $S$ be another continuous group action of $G$ on $X$ with $d_{A}(S, T) \leq \delta$. Define a set-valued map $H$ of $X$ by 


$$
H(x)=\bigcap_{g \in G} T_{g}^{-1}\left(B\left[S_{g}(x), \varepsilon^{\prime}\right]\right), \quad \forall x \in X
$$

Clearly, $H$ is a compact-valued map.

Let us prove that $\operatorname{Dom}(H)$ is measurable. Take a sequence $x_{n} \in \operatorname{Dom}(H)$ conversing to some $x \in X$. Since $x_{n} \in \operatorname{Dom}(H)$, we can choose a sequence $y_{n} \in X$ such that

$$
d\left(T_{g} y_{n}, S_{g} x_{n}\right) \leq \varepsilon^{\prime}, \forall g \in G, \forall n \in \mathbb{Z}
$$

Since $X$ is compact, we can assume that $y_{n} \rightarrow y$ for some $y \in X$. By fixing $g \in G$ and letting $n \rightarrow \infty$, we have $d\left(T_{g} y, S_{g} x\right) \leq \varepsilon^{\prime}$ for every $g \in G$. This means that $y \in H(x)$, and so $x \in \operatorname{Dom}(H)$. Consequently $\operatorname{Dom}(H)$ is measurable.

Next we show that $\mu(X \backslash \operatorname{Dom}(H))=0$. Since $d_{A}(T, S) \leq \delta$, the $S$-orbit $\left(S_{g}(x)\right)_{g \in G}$ of every point $x \in X$ is a $\delta$-pseudo orbit for $T$ with respect to $A$. By taking $S_{e}(x)=x \in B$ we have that such an $S$-orbit is through $B$, hence it can be $\varepsilon^{\prime}$-traced by some point in $X$. It follows that there is $y \in X$ such that $d\left(T_{g} y, S_{g} x\right) \leq \varepsilon^{\prime}$ for every $g \in G$ and $x \in X$. From this we have $H(x) \neq \emptyset$ for every $x \in B$. Then $B \subset \operatorname{Dom}(H)$ and so $\mu(X \backslash \operatorname{Dom}(H)) \leq \mu(X \backslash B)=0$.

To show that $H$ is upper semicontinuous, fix $x \in \operatorname{Dom}(H)$ and an open neighborhood $O$ of $H(x)$. Define a map $H: X \rightarrow 2^{X}$ by

$$
H(y)=\bigcap_{m=0}^{\infty} H_{m}(y),
$$

where $H_{m}(y)=\bigcap_{g \in B(m)} T_{g^{-1}}\left(B\left[S_{g}(y), \varepsilon^{\prime}\right]\right), B(m)=\left\{g \in G \mid l_{A}(g) \leq m\right\}$, and $l_{A}(g)$ is the word length metric on $G$ induced by $A$. Note that each $H_{m}(y)$ is compact and $H_{m+1}(y) \subset H_{m}(y)$ for $m$ and $y \in X$. By taking $y=x$, we have $H_{m}(x) \subset O$. We claim that there is $\eta>0$ such that $H_{m}(y) \subset O$ whenever $d(x, y)<\eta$. Otherwise, there are sequences $y_{g_{k}} \rightarrow x$ and $z_{g_{k}} \in H_{m}\left(y_{g_{k}}\right) \backslash O$ for all $g_{k}$ with $l_{A}\left(g_{k}\right)=k$. Since $X$ is compact, we can assume that $z_{g_{k}} \rightarrow z$ for some $z \in X$. Clearly, $z \notin O$. However $z_{g_{k}} \in H_{m}\left(y_{g_{k}}\right)$ so $d\left(T_{g}\left(z_{g_{k}}\right), S_{g}\left(y_{g_{k}}\right) \leq \varepsilon^{\prime}\right.$ for $g \in B(m)$ and all $g_{k}$ with $l_{A}\left(g_{k}\right)=k$. Then we get,

$$
\begin{aligned}
d\left(T_{g} z, S_{g} x\right) & \leq d\left(T_{g}(z), T_{g}\left(z_{g_{k}}\right)\right)+d\left(T_{g}\left(z_{g_{k}}\right), S_{g}\left(y_{g_{k}}\right)\right)+d\left(S_{g}\left(y_{g_{k}}\right), S_{g}(x)\right) \\
& \leq d\left(T_{g}(z), T_{g}\left(z_{g_{k}}\right)\right)+d\left(S_{g}\left(y_{g_{k}}\right), S_{g}(x)\right)+\varepsilon^{\prime},
\end{aligned}
$$

for $g \in B(m)$. Letting $k \rightarrow \infty$ we get $d\left(T_{g}(z), S_{g}(x)\right) \leq \varepsilon^{\prime}$ for any $g \in B(m)$, so $z \in H_{m}(x)$. Since $z \notin O$ and $H_{m}(x) \subset O$. We arrive at a contradiction. Consequently, if $d(x, y)<\eta$ then $H(y) \subset H_{m}(y) \subset O$, and so $H$ is uppersemicontinuous.

Now we prove $\mu \circ H=0$ and $d\left(H, I d_{X}\right) \leq \varepsilon$. Take $x \in X$ and $y \in H(x)$. If $z \in H(x)$, we have $d\left(T_{g} z, S_{g} x\right) \leq \varepsilon^{\prime}$ for $g \in G$. Since $y \in H(x)$ we have $d\left(T_{g} y, S_{g} x\right) \leq$ $\varepsilon^{\prime}$. This implies $d\left(T_{g} y, T_{g} z\right) \leq 2 \varepsilon^{\prime}$ for $g \in G$. Hence $z \in \Gamma_{c}^{T}(y)$ and $H(x) \subset \Gamma_{c}^{T}(y)$. 
Therefore, $\mu(H(x)) \leq \mu\left(\Gamma_{c}^{T}(y)\right)=0$. Since $c$ is an expansive constant of $\mu$, we have $\mu \circ H=0$. It follows from the definition of $H$ that $H(x) \subset B\left[x, \varepsilon^{\prime}\right]$. Since $\varepsilon^{\prime}<\varepsilon$, we have $d\left(H, I d_{X}\right) \leq \varepsilon$.

Finally, we prove $T_{g} \circ H=H \circ S_{g}$ for $g \in G$. If $x \in \operatorname{Dom}(H)$, then we have

$$
\begin{aligned}
T_{g}(H(x)) & =T_{g}\left(\bigcap_{h \in G} T_{h}^{-1}\left(B\left[S_{h}(x), \varepsilon^{\prime}\right]\right)=\bigcap_{h \in G} T_{g h^{-1}}\left(B\left[S_{h}(x), \varepsilon^{\prime}\right]\right)\right. \\
& =\bigcap_{g_{1} \in G} T_{g_{1}}^{-1}\left(B\left[S_{g_{1}}(x), \varepsilon^{\prime}\right]\right)=\bigcap_{g_{1} \in G} T_{g_{1}}^{-1}\left(B\left[S_{g_{1}}\left(S_{g} x\right), \varepsilon^{\prime}\right]\right)=H\left(S_{g}(x)\right),
\end{aligned}
$$

where $g_{1}=h g^{-1}$. So $\operatorname{Dom}(H)$ is $S$-invariant and $T_{g} \circ H=H \circ S_{g}$ in $\operatorname{Dom}(H)$. It follows that $S_{g}(x) \notin \operatorname{Dom}(H)$ if $x \notin \operatorname{Dom}(H)$. Hence we obtain $T_{g}(H(x))=\emptyset=$ $H\left(S_{g}(x)\right)$, and so $T_{g} \circ H=H \circ S_{g}$ on $X \backslash \operatorname{Dom}(H)$. This completes the proof.

Next we will provide a class of measure topologically stable actions, and study the dynamical properties of those actions. First, we recall the definition of nilpotent group. Let $G$ be a countable group. The lower central series of $G$ is the sequence $\left\{G_{i}\right\}_{i \geq 0}$ of subgroups of $G$ defined by $G_{0}=G$ and $G_{i+1}=\left[G_{i}, G\right]$, where $\left[G_{i}, G\right]$ is the subgroup of $G$ generated by all commutators $[a, b]:=a b a^{-1} b^{-1}, a \in G_{i}, b \in G$. The group $G$ is said to be nilpotent if there exists $n \geq 0$ such that $G_{n}=\left\{e_{G}\right\}$. The such smallest $n$ is called the nilpotent degree of $G$.

We say that $\mu \in \mathcal{M}(X)$ is invariant for group action $T$ if $\mu(B)=\mu\left(T_{g}(B)\right)$ for any Borel set $B$ and $g \in G$. We denote by $\mathcal{M}_{T}(X)$ and $\mathcal{M}_{T}^{*}(X)$ the set of all invariant Borel probability measures and the set of all invariant nonatomic Borel probability measures on $X$, respectively. We say that a group action $T$ has invariant measure shadowing property if and only if $T$ has $\mu$-shadowing for all $\mu \in \mathcal{M}_{T}^{*}(X)$.

Theorem 3.3. Let $G$ be a finitely generated virtually nilpotent group, i.e., there exists a nilpotent normal subgroup $H$ of $G$ with finite index. Let $T$ be a continuous group action of $G$ on a compact metric space $X$. Suppose $T_{g}$ is expansive and has the invariant measure shadowing property for some $g \in G$, then the action $T$ is invariant measure topologically stable.

To prove the above theorem, we need some following lemmas.

Lemma 3.4. Let $T \in \operatorname{Act}(G, X)$ and $\mu \in \mathcal{M}(X)$. If $T$ is expansive with expansive constant $\eta$ and has the $\mu$-shadowing property. Let $\varepsilon<\frac{\eta}{2}$, then every $\delta$-pseudo orbit of $T$ as in the definition of $\mu$-shadowing property with respect to $\varepsilon$ has a unique shadowing point in $X$.

Proof. For any $\varepsilon>0$ choose $\delta>0$ and a Borel set $B$ with $\mu(B)=1$ as in the definition of $\mu$-shadowing property. Let $\left\{x_{g}\right\}_{g \in G}$ be a $\delta$-pseudo orbit of $T$ through $B$ 
and let $\eta$ be an expansive constant with $\eta>2 \varepsilon$. Assume that $x, y$ be two points that $\varepsilon$-traced $\left\{x_{g}\right\}_{g \in G}$. Then one has

$$
d\left(T_{g} x, T_{g} y\right) \leq d\left(T_{g} x, x_{g}\right)+d\left(x_{g}, T_{g} y\right)<\varepsilon+\varepsilon<\eta
$$

for every $g \in G$. Since $T$ is expansive, we have $x=y$.

Lemma 3.5. Let $G$ be a finitely generated group and $H$ be a finitely generated normal subgroup of $G$. Let $T \in \operatorname{Act}(G, X)$ and $\mu \in \mathcal{M}_{T}(X)$. If the restriction map $T_{H}$ on $H \times X$ is expansive and has the $\mu$-shadowing property, then $T$ has the $\mu$-shadowing property.

Proof. Let $\mu \in \mathcal{M}_{T}(X)$ and $A_{H}$ be a symmetric finitely generating set of $H$. We can add more elements to $A_{H}$ to get a finitely generating set $A$ of $G$. Let $c$ be the expansive constant of $T_{H}$. Since $X$ is compact and $A$ is finite, there exists $0<\eta<\frac{c}{3}$ such that $d\left(T_{b} x, T_{b} y\right)<\frac{c}{3}$ for every $b \in A$ and all $x, y \in X$ with $d(x, y)<\eta$. Let $\varepsilon>0$ be a constant with $\varepsilon<\eta$, since $T_{H}$ has the $\mu$-shadowing property, we can choose $0<\delta<\varepsilon$ and Borel set $B_{H}$ with $\mu\left(X \backslash B_{H}\right)=0$ such that every $\delta$-pseudo orbit of $T_{H}$ with respect to $A_{H}$ through $B_{H}$ is $\frac{\varepsilon}{2}$-traced by some point of $X$. Let

$$
B=B_{H} \cap \operatorname{supp}(\mu)
$$

First, we claim that $\mu(B)=1$. Indeed, by contradiction, we suppose that $0 \leq \mu(B)<$ 1. Let $B^{\prime}=B_{H} \bigcap(\operatorname{supp}(\mu))^{c}$, then $B_{H}=B^{\prime} \cup B$ and hence $\mu\left(B^{\prime}\right)>0$. For any $x \in B^{\prime}, x \notin \operatorname{supp}(\mu)$, there exists a neighborhood $U_{x}$ such that $\mu\left(U_{x}\right)=0$. Then

$$
B^{\prime} \subset \bigcup_{x \in B^{\prime}} U_{x}, \text { so } \mu\left(\bigcup_{x \in B^{\prime}} U_{x}\right)>\mu\left(B^{\prime}\right)>0 .
$$

However, $\left(\bigcup_{x \in B^{\prime}} U_{x}\right) \bigcap \operatorname{supp}(\mu)=\emptyset$ and then $\mu\left(\bigcup_{x \in B^{\prime}} U_{x}\right)=0$. The contradiction shows that $\mu(B)=1$. Let $\left\{x_{g}\right\}_{g \in G}$ be a $\frac{\delta}{2}$-pseudo orbit with respect to $A$ through $B$. For every $g \in G$, the sequence $\left\{x_{h g}\right\}_{h \in H}$ is a $\frac{\delta}{2}$-pseudo orbit of $T_{H}$ with respect to $A_{H}$. Since $\mu$ is invariant for $T$ and $B$ is a subset of $\operatorname{supp}(\mu)$ with $\mu(B)=1, B$ is dense in $\operatorname{supp}(\mu)$. For $\frac{\delta}{2}>0$, we choose $0<\eta^{\prime}<\frac{\delta}{2}$ and $y_{g} \in B$ such that $d\left(y_{g}, x_{g}\right)<\eta^{\prime}<\frac{\delta}{2}$ and $d\left(T_{a} x_{g}, T_{a} y_{g}\right)<\frac{\delta}{2}$ for every $g \in G, a \in A_{H}$. We define a new sequence $\left\{y_{h g}\right\}_{h \in H}$ by

$$
y_{h g}= \begin{cases}y_{g} & h=e, \\ x_{h g} & h \neq e .\end{cases}
$$

for every $g \in G$ such that

$$
d\left(T_{a} y_{h g}, y_{a h g}\right)= \begin{cases}d\left(T_{a} x_{a^{-1}}, y_{g}\right) & h=a^{-1} \\ d\left(T_{a} y_{g}, x_{a g}\right) & h=e, \\ d\left(T_{a} x_{h g}, x_{a h g}\right) & h \neq e, a^{-1} .\end{cases}
$$


Then,

$$
\begin{aligned}
& d\left(T_{a} x_{a^{-1} g}, y_{g}\right) \leq d\left(T_{a} x_{a^{-1} g}, x_{a a^{-1} g}\right)+d\left(x_{g}, y_{g}\right)<\frac{\delta}{2}+\frac{\delta}{2}=\delta, \\
& d\left(T_{a} y_{g}, x_{a g}\right) \leq d\left(T_{a} y_{g}, T_{a} x_{g}\right)+d\left(T_{a} x_{g}, x_{a g}\right)<\frac{\delta}{2}+\frac{\delta}{2}=\delta, \\
& d\left(T_{a} x_{h g}, x_{a h g}\right)<\delta .
\end{aligned}
$$

So, $\left\{y_{h g}\right\}_{h \in H}$ is a $\delta$-pseudo orbit of $T_{H}$ with respect to $A_{H}$ through $B$ hence through $B_{H}$. Since $T_{H}$ is expansive, by Lemma 3.4 there exists a unique point $z_{g} \in X$ such that

$$
d\left(y_{h g}, T_{h}\left(z_{g}\right)\right)<\frac{\varepsilon}{2} \text { for every } h \in H .
$$

Now we prove that $z_{g}=T_{g} z_{e}$ for every $g \in G$. Fix $g \in G$ and $b \in A$. For each $h \in H$, there exists $h^{\prime} \in H$ such that $h b=b h^{\prime}$ by $H$ is a normal subgroup of $G$. Then we have

$$
d\left(y_{b h^{\prime} g}, T_{h} z_{b g}\right)=d\left(y_{h b g}, T_{h} z_{b g}\right)<\frac{\varepsilon}{2}
$$

and

$$
d\left(T_{b} y_{h^{\prime} g}, T_{h} T_{b} z_{g}\right)=d\left(T_{b} y_{h^{\prime} g}, T_{b} T_{h^{\prime}} z_{g}\right)<\frac{c}{3}
$$

Hence we get

$$
\begin{aligned}
d\left(T_{h} z_{b g}, T_{h} T_{b} z_{g}\right) & \leq d\left(T_{h} z_{b g}, y_{b h^{\prime} g}\right)+d\left(y_{b h^{\prime} g}, T_{b} y_{h^{\prime} g}\right)+d\left(T_{b} y_{h^{\prime} g}, T_{h} T_{b} z_{g}\right) \\
& \leq \frac{\varepsilon}{2}+\delta+\frac{c}{3}<c .
\end{aligned}
$$

Since $T_{H}$ is expansive, we have $T_{b} z_{g}=z_{b g}$ for every $b \in A$. As $A$ is symmetric generating set of $G$, we get $z_{g}=T_{g} z_{e}$ for every $g \in G$. On the other hand, by applying $h=e$ for $d\left(y_{h g}, T_{h}\left(z_{g}\right)\right)<\frac{\varepsilon}{2}$, we have $d\left(y_{g}, z_{g}\right)<\frac{\varepsilon}{2}$ and then

$$
d\left(x_{g}, z_{g}\right)=d\left(x_{g}, y_{g}\right)+d\left(y_{g}, z_{g}\right)<\frac{\delta}{2}+\frac{\varepsilon}{2}<\varepsilon
$$

for every $g \in G$. So $d\left(T_{g} z_{e}, x_{g}\right)=d\left(z_{g}, x_{g}\right)<\varepsilon$ for all $g \in G$ thus $T$ has the $\mu$-shadowing property.

Recall that for an action $T \in \operatorname{Act}(G, X), T_{g}$ is a homeomorphism for every $g \in G$. We call that $T_{g}$ has the $\mu$-shadowing property if for any $\varepsilon>0$ there are $\delta>0$ and a Borel set $B$ with $\mu(X \backslash B)=0$ such that for any $\delta$-pseudo orbit $\left\{x_{n}\right\}_{n \in \mathbb{N}}$ of $T_{g}$ through $B$ is $\varepsilon$-traced by some point in $X$.

Lemma 3.6. Let $T$ be a continuous action on a compact metric space $X$. Then for any $g \in G, T_{g}$ has the $\mu$-shadowing property then $T_{g^{n}}$ has the $\mu$-shadowing property for any $n \in \mathbb{Z}$. 
Proof. For any $\varepsilon>0$, choose $\delta_{1}>0$ and a Borel set $B$ corresponding to the definition of $\mu$-shadowing property with respect to $T_{g}$. For $\varepsilon>0$ choose $0<\delta_{n}<\delta_{1}$ such that $\left\{x_{n}\right\}_{n \in \mathbb{N}}$ is a $\delta_{n}$-pseudo orbit of $T_{g_{n}}$ through $B$. Now we show that the $\delta_{n}$-pseudo orbit $\left\{x_{n}\right\}_{n \in \mathbb{N}}$ of $T_{g_{n}}$ through $B$ is also a $\delta_{1}$-pseudo orbit of $T_{g}$ through $B$. Define a sequence $\left\{y_{m}\right\}_{m \in \mathbb{N}}$ as $y_{l n}=x_{l}$ for all $l \in \mathbb{Z}, y_{l n+i}=T_{g^{i}} x_{l}$ for $1 \leq i \leq n-1$ and for all $l \in \mathbb{Z}$. Since $d\left(T_{g^{n}} x_{0}, x_{1}\right)<\delta_{n}, d\left(T_{g} y_{n-1}, y_{n}\right)<\delta_{n}$, it means that $\left\{y_{n}\right\}_{n \in \mathbb{N}}$ is a $\delta_{1}$-pseudo orbit of $T_{g}$ through $B$. Hence there is $y \in X$ such that $d\left(\left(T_{g}\right)^{k}, y_{k}\right)<\varepsilon$, so for $k=\ln$ where $l \in \mathbb{N}$, we have $d\left(\left(T_{g}\right)^{l n} y, y_{l n}\right)=d\left(\left(T_{g}^{n}\right)^{l} y, x_{l}\right)<\varepsilon$ so complete the proof.

The following lemma whose proof is similar to that of Lemmas 2.13 in [4]. For satety, we give detailed proof.

Lemma 3.7. Let $G$ be a finitely generated virtually nilpotent group and $T$ be a continuous action of $G$ on a compact metric space $X$. If there exists $g \in G$ such that $T_{g}$ is expansive and has the $\mu$-shadowing property for some Borel measure $\mu \in \mathcal{M}_{T}^{*}(X)$ on $X$, then $T$ has the $\mu$-shadowing property.

Proof. We prove by induction on the nilpotent degree $n$ of $G$. If $n=1$, then the group $G$ is abelian and hence $H=\langle g\rangle$ is a normal subgroup of $G$. Thus, by the previous two lemmas $T$ has the $\mu$-shadowing property. Let $n>1$ and assume that this lemma holds for each nilpotent group with nilpotent degree less than or equal to $n-1$. Put $G_{1}=[G, G]$ and $K=<G_{1}, g>$, then $K$ has the nilpotent degree at most $n-1$ by Proposition $2(\mathrm{~N} 2)$ in [7]. It is known that $G_{1}$ is finitely generated by Lemma 6.8.4 in [5] and hence $K$ is finitely generated. Thus from the assumption, $T_{K}$ has the $\mu$-shadowing property. Since $T_{g}$ is expansive and $g \in K, T_{K}$ is expansive. As $K$ is a normal subgroup of $G$ by Proposition 2(N1) in [7], applying Lemma 3.5, we get the result.

Proof of Theorem 3.3. Let $H$ be a nilpotent normal subgroup of $G$ with finite index. Then $H$ is finitely generated by Proposition 6.6.2 in [5]. Since $H$ has finite index in $G$, there exists $n \in \mathbb{N}$ such that $g^{n} \in H$ for any $g \in G$. Since $T_{g}$ is an expansive homeomorphism and $g^{n} \in H$, then $T_{g^{n}}=T_{g}{ }^{n}$ is also expansive by Theorem 2.2.4 in [1] and also has the $\mu$-shadowing property by the Lemma 3.6. Thus from Lemma 3.7, $T_{H}$ has the $\mu$-shadowing property. Since $T_{g^{n}}$ is expansive, $T_{H}$ is expansive so is $T$, hence $T$ is $\mu$-expansive for every $\mu \in \mathcal{M}^{*}(X)$. Therefore, applying Theorem 3.2 and Lemma 3.5, $T$ is $\mu$-topologically stable.

In the following theorem, we provide a class of group actions which do not have any topologically stable measures. For this, we first recall that an action $T \in \operatorname{Act}(G, X)$ is 
minimal if every $T$-orbit $\left\{T_{g} x \mid g \in G\right\}(x \in X)$ is dense in $X$, and $T$ is called periodic if $\left\{T_{g} x \mid g \in G\right\}(x \in X)$ is finite.

Theorem 3.8. A minimal action $T \in \operatorname{Act}(G, X)$ approximated by periodic actions is not $\mu$-topologically stable for any $\mu \in \mathcal{M}(X)$.

Proof. Let $T \in \operatorname{Act}(G, X)$ be a minimal action which can be approximated by periodic actions, and suppose there exists a Borel measure $\mu \in \mathcal{M}(X)$ which is topologically stable with respect to $T$. For $\varepsilon=1$, choose a constant $\delta>0$ corresponding to $\varepsilon$ by the definiton of $\mu$-topological stability of $T$. Choose a periodic action $S \in \operatorname{Act}(G, X)$ with $d_{A}(T, S) \leq \delta$ for some fintely generating set $A$. Then we have that $\mu(\operatorname{Per}(S))=0$. Suppose not. Then, by the topological stability of $\mu$ with respect to $T$, there is an upper semicontinuous compact-valued map $H$ with measurable domain $X$ satisfying $\mu(X \backslash \operatorname{Dom}(H))=0$ and $\mu \circ H=0$ and $T_{g} \circ H=H \circ S_{g}$ for every $g \in G$. Since $\mu(\operatorname{Per}(S))>0$ and $\mu(X \backslash \operatorname{Dom}(H))=0$, there exists a point $x \in \operatorname{Per}(S) \bigcap \operatorname{Dom}(H)$. Since $x$ is a periodic point of $S$, we can easily show that $\Lambda=\bigcup_{g \in G} H\left(S_{g} x\right)$ is both compact and invariant. As $\mu \circ H=0$, we also have $\mu(\Lambda)=0$. As $H(x) \neq \emptyset$ we can choose $y \in H(x)$. Since $H(x) \subset \Lambda$ we have $y \in \Lambda$. But $T$ is minimal so the $T$-orbit of $y$ is dense in $X$. As $\Lambda$ is compact and invariant we obtain that the closure of such an $T$-orbit is contained in $\Lambda$. Hence we reach that $X=\Lambda$, and so $\mu(X)=\mu(\Lambda)=0$. This shows that $\mu(\operatorname{Per}(S))=0$. On the other hand, since $S$ is periodic, we have $\mu(\operatorname{Per}(S))=1$. Consequently the contradiction completes the proof.

Finally we show that any action $T \in \operatorname{Act}(G, X)$ with a topologically stable measure, and which can be approximated by minimal actions is recurrsive, i.e., $\Omega(T)=X$. Recall that a point $x \in X$ is nonwandering for $T$ if, for any neighborhood $U$ of $x$ there is $g \in G \backslash\{e\}$ such that $T_{g}(U) \bigcap U \neq \emptyset$. The set of all such points is denoted by $\Omega(T)$.

Lemma 3.9. Let $A$ be a finitely generating set of $G$, and let $T \in A c t(G, X)$ be and action with a topologically stable measure $\mu$. Then for any $\varepsilon>0$, there is $\delta>0$ and a subset $B$ of $X$ with $\mu(X \backslash B)=0$ such that if $d_{A}(T, S)<\delta$ then every $S$-orbit of $x$ can be $\varepsilon$-traced in $T$ for all $x \in B$.

Proof. Fix $\varepsilon>0$ and let $\delta>0$ be given by the $\mu$-topological stability of $T$. Take a continuous action $S$ with $d_{A}(T, S) \leq \delta$. For this $S$ let $H$ be as in the definition of $\mu$ topological stability of $T$ with $\mu(X \backslash \operatorname{Dom}(H))=0$. We choose $B=\operatorname{Dom}(H)$. Then for any $x \in B$ we have $y \in H(x)$ and hence $T_{g} y \in T_{g}(H(x))=H\left(S_{g} x\right) \subset B\left[S_{g} x, \varepsilon\right]$ and so $d\left(T_{g} y, S_{g} x\right) \leq \varepsilon$ for all $g \in G$ completing the proof.

Theorem 3.10. Let $T \in \operatorname{Act}(G, X)$ be an action which can be approximated by 
minimal actions. If $T$ has a topologically stable measure, then $\Omega(T)=X$.

Proof. Let $\mu$ be a topologically stable measure of $T$. Fix $\varepsilon>0$ and for any $z \in X$ we show that $z \in \Omega(T)$. For the given $\varepsilon$ and $\mu$, by the previous lemma there are $\delta>0$ and $B$ with $\mu(B)=1$ such that every $S$-orbit of any $x \in B$ with $d_{A}(T, S)<\delta$ is $\frac{\varepsilon}{2}$-traced in $T$. By hypothesis there is a minimal action $S \in \operatorname{Act}(G, X)$ with $d_{A}(T, S) \leq \delta$ and for any $x \in B$, the $S$-orbit $\left\{S_{g} x \mid g \in G\right\}$ of $x$ is dense in $X$. Since $x \in B$, there is $y \in X$ such that $d\left(T_{g} y, S_{g} x\right)<\frac{\varepsilon}{2}$ for any $g \in G$. Since $\overline{\left\{S_{g} x \mid g \in G\right\}}=X$, there is a sequence $\left(g_{n}\right)_{n \in \mathbb{N}} \subset G$ with $l_{A}\left(g_{n}\right)=n$ such that $\lim _{n \rightarrow \infty} S_{g_{n}}(x)=z$ for some $z \in X$. Then there is $m \in \mathbb{N}$ such that $d\left(S_{g_{n}}(x), z\right)<\frac{\varepsilon}{2}$ for all $g_{n}$ with $l_{a}\left(g_{n}\right) \geq m$. Since $X$ is compact we can assume that $T_{g_{n}} y \rightarrow w$ for some $w \in X$. Let $U$ be a neighborhood of $w$ then there exists $g_{n}, g_{n+1} \in G$ such that $T_{g_{n}} y, T_{g_{n+1}} y \in U$ for $n$ large enough. Choose $h=g_{n} g_{n+1}^{-1}$. Then $T_{h} T_{g_{n+1}} y=T_{g_{n}} y \in U$ hence $T_{g_{n+1}} y \in T_{h^{-1}} U$, and we have $w \in \Omega(T)$. Since

$$
\begin{aligned}
d\left(z, T_{g_{n}} y\right) & \leq d\left(z, S_{g_{n}} x\right)+d\left(S_{g_{n}} x, T_{g_{n}} y\right) \\
& <\varepsilon / 2+\varepsilon / 2=\varepsilon
\end{aligned}
$$

for all $g_{n} \in G$ with $l_{a}\left(g_{n}\right) \geq m$. By letting $n \rightarrow \infty$ we have $d(z, w) \leq \varepsilon$. Since $w \in \Omega(T)$ we conclude that

$$
d(z, \Omega(T))=\inf _{w \in \Omega(T)} d(z, w) \leq \varepsilon
$$

As $z, \varepsilon$ are arbitrary, we conclude that $\Omega(T)$ is dense in $X$. As $\Omega(T)$ is closed, we obtain $\Omega(T)=X$ and complete the proof.

\section{REFERENCES}

[1] N. Aoki and K. Hiraide, Topologically Theory of Dynamical Systems, NorthHolland Mathematical Library, 52, North-Holland Publishing Co., Amsterdam, 1994.

[2] A. Arbieto and C.A Morales, Some properties of positive entropy maps, Ergodic Theory Dynam. Systems, 34 (2014), no. 3, 765-776.

[3] A. Artigue and D. Carrasco-Olivera, A note on measure-expansive diffeomorphisms, J. Math. Anal. Appl., 428 (2015), no. 1, 713-716.

[4] N.P Chung and K. Lee, Topological stability and pseudo-orbit tracing property of group actions, To appear in Proc. Amer. Math. Soc..

[5] M. Coornaert and T.C. Silberstein, Cellular Automata and Groups, Springer Monographs in Mathematics, Springer-Verlag, Berlin, 2010. 
[6] K. Lee and C.A Morales, Topological stability and pseudo-orbit tracing property for expansive measures, J. Differential Equations, 262 (2017), 3467-3487.

[7] A.V. Osipov and S.B. Tikhomirov, Shadowing for actions of some finitely generated groups, Dyn. Syst., 29 (2014), 337-351.

[8] M.J. Pacifico and J.L. Vietez, On measure expansive diffeomorphisms, Proc. Amer. Math. Soc., 143 (2015), no. 2, 811-819.

[9] S.Y Pilyugin, Inverse shadowing in group action, Dyn. Syst., 32 (2017), 198-210.

[10] S.Y. Pilyugin and S.B. Tikhomirov, Shadowing in actions of some Abelian groups, Fund. Math., 179 (2003), 83-96.

[11] K. Sakai, N. Sumi, and K. Yamamoto, Measure-expansive diffeomorphisms, J. Math. Anal. Appl., 414 (2014), no. 2, 546-552.

[12] P. Walters, On the pseudo orbit tracing property and its relationship to stability, Lecture Notes in Math., 668 (1978), 231-244.

[13] K. Yano, Topologically stable homeomorphisms of the circle, Nagoya Math. J., 79 (1980), 145- 149. 
